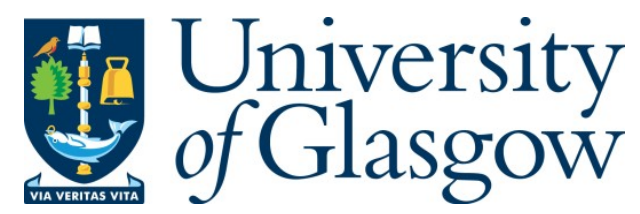

O'Shea, K.J., McGrouther, D., Ferguson, C.A., Jungbauer, M., Hühn, S., Moshnyaga, V., and MacLaren, D.A. (2014) Fabrication of high quality plan-view TEM specimens using the focused ion beam. Micron, 66 . pp. 915. ISSN 0968-4328

Copyright (C) 2014 Elsevier Ltd.

http://eprints.gla.ac.uk/93381/

Deposited on: 2 May 2014

Enlighten - Research publications by members of the University of Glasgow http://eprints.gla.ac.uk 


\title{
Fabrication of high quality plan-view TEM specimens using the focused ion beam
}

\author{
K.J. O'Shea ${ }^{\mathrm{a}}$, D. McGrouther ${ }^{\mathrm{a}}$, C. A. Ferguson ${ }^{\mathrm{a}}$, M. Jungbauer ${ }^{\mathrm{b}}$, S. Hühn ${ }^{\mathrm{b}}$, V. \\ Moshnyaga $^{\mathrm{b}}$ and D.A. MacLaren ${ }^{\mathrm{a}}$ \\ ${ }^{a}$ SUPA, School of Physics and Astronomy, University of Glasgow, G12 8QQ, UK \\ ${ }^{b}$ Univ. Gottingen, Inst Phys 1, Friedrich Hund Pl 1, D-37077 Gottingen, Germany
}

\begin{abstract}
We describe a technique using a focused ion beam instrument to fabricate high quality plan-view specimens for transmission electron microscopy studies. The technique is simple, site-specific and is capable of fabricating multiple large, $>100 \mu \mathrm{m}^{2}$ electron transparent windows within epitaxially-grown thin films. A film of $\mathrm{La}_{0.67} \mathrm{Sr}_{0.33} \mathrm{MnO}_{3}$ is used to demonstrate the technique and its structural and functional properties are surveyed by high resolution imaging, electron spectroscopy, atomic force microscopy and Lorentz electron microscopy. The window is demonstrated to have good thickness uniformity and a low defect density that does not impair the film's Curie temperature. The technique will enable the study of in-plane structural and functional properties of a variety of epitaxial thin film systems.
\end{abstract}

Keywords: FIB, plan-view, TEM, AFM, epitaxial.

\section{Introduction}

The focused ion beam (FIB) instrument is an extremely versatile tool for the preparation of high quality specimens for transmission electron microscopy (TEM) [1-3], for rapid lithographic device prototyping [4-6] and for local modification of material properties [7-9]. FIB preparation of cross-sectional specimens for TEM is now commonplace [10] and is applicable to almost arbitrary materials, including polymers [11], biomaterials [12] and ceramics [13]. A major advantage is the unrivalled site-specificity offered by dual-beam FIB systems, which simultaneously image a sample with an electron beam whilst using an ion beam (most commonly $\mathrm{Ga}^{+}$) to cut out a thin, micron-scale lamella from a bulk specimen [14]. Popular FIB techniques are well-suited to samples that can be cross-sectioned; i.e. where the structural features of interest extend several tens of nanometres in at least one dimension that can be aligned to lie perpendicular to the plane of the final TEM specimen. In these cases the tolerance on positional accuracy is eased and crosssection samples are routinely prepared to enable analysis of, for example, grain boundaries and thin film interfaces. However, these techniques are less suited to planview TEM studies, where the plane of the electron-transparent lamella may need to be aligned to include nanomaterials of comparable thickness to that of the lamella itself. One example of where plan-view samples are particularly important is in the characterisation of features within the plane of a thin film. Rather than the limited field of view offered by a cross-section through the film, it is preferable to align the plane of the film perpendicular to the TEM electron beam - i.e. in plan view - in order to assess areal densities of defects, or to map strain or functional properties such 
as ferromagnetism. The latter could be achieved, for example, by imaging the response of patterned elements within the film to an external electric or magnetic field, similar to studies of amorphous materials described elsewhere, using either the microscope's own magnetic field or that created by electrical connections within the sample rod [15]. A difficulty arises in the preparation of plan-view samples because it is challenging to align and retain a wide area of undamaged thin film within the plane of the TEM lamella. Previous top-down preparations of plan-view specimens have used chemical etching [16,17], Ar-ion milling [18,19] and tripod polishing [20]. However, etching protocols require material-specific, highly selective etchants whilst milling and polishing provide very limited site-specificity. In some cases, the thin film of interest can instead be deposited directly onto an electron-transparent membrane such as amorphous silicon nitride [21] or single-crystalline GaAs [22] but these substrates do not provide a widely-applicable protocol since they are still limited to the growth of amorphous, polycrystalline or specific lattice-matched materials. It would therefore be useful to employ the advantages of FIB techniques, and although a small number are mentioned in the literature we have found that they can be unreliable. These techniques tend to conclude with sequential ion-polishing of either side of the lamella, where it can be difficult to ensure that the material of interest is retained within a lamella that may only be a few tens of nanometres thick. As a consequence, recent reports that successfully employ a FIB for site-selective preparation of plan-view TEM specimens [23-31] often employ intricate [23] or multi-step processes, for example the use of a micromanipulator $[24,28]$, that can increase the risk of specimen loss or damage. In this manuscript we propose a simpler approach that derives from previous 'H-bar' techniques [24] and is appropriate for the fabrication of plan-view specimens from epitaxially-grown thin films. Notably, we use a combination of electron microscopy techniques and atomic force microscopy to assess the quality of lamellae produced. We demonstrate, using a ferromagnetic perovskite oxide epitaxial film, a FIB-based preparation of plan-view samples that is widely applicable, preserves the substrate/film epitaxy and imparts minimal damage to the region of interest. Furthermore, this approach enables multiple site-specific plan-view specimens to be fabricated from one polished film, which is challenging using conventional techniques.

\section{Specimen pre-treatment}

The thin film used in this study is the perovskite $\mathrm{La}_{0.67} \mathrm{Sr}_{0.33} \mathrm{MnO}_{3}$ (LSMO) grown on $\mathrm{SrTiO}_{3}$ (STO), one of a number of complex oxide heterostructures of current interest for device fabrication and emergent interfacial phenomena [32, 33]. LSMO is a ferromagnetic half metal with a Curie temperature, $\mathrm{T}_{\mathrm{C}}$, around $370 \mathrm{~K}$, which is useful in the context of this study since $\mathrm{T}_{\mathrm{c}}$ is known to be depressed by structural defects and so serves as a simple measure of film quality [34]. LSMO was deposited as a $120 \mathrm{~nm}$ thick film onto a $500 \mu \mathrm{m}$ thick $\mathrm{SrTiO}_{3}(111)$ (STO) substrate by metalorganic aerosol deposition (MAD) [35], a technique that we have found to be very reliable for the production of films of a high Curie temperature, suggesting a low defect density. The (111) STO substrate orientation is of particular interest as a result of proposed enhanced structural and electronic coupling at the surface [36]. Cross-sectional TEM imaging (not shown here) shows there to be high quality epitaxial growth of the LSMO film on STO. Prior to FIB work, approximately $470 \mu \mathrm{m}$ of material was removed from the sample's underside as follows. The sample was fixed, with the LSMO film face-down, onto a specimen mount using Kemdent ${ }^{\circledR}$ wax to protect the 
LSMO. It was then thinned to $\sim 30 \mu \mathrm{m}$ using a Gatan® Disc Grinder and diamond paper, using progressively finer grades ( $30 \mu \mathrm{m}$ to $0.5 \mu \mathrm{m}$ grit). The sample was then mounted onto a slotted TEM $\mathrm{Cu}$ grid that had been partially cut away using an epoxy adhesive (Araldite ${ }^{\circledR}$, cured at $55^{\circ} \mathrm{C}$ for 1 hour), similar to the process sketched in Ref. [1]. It was then placed onto a hotplate to melt the wax and release the original mount. Wax residue was removed using acetone and the specimen was cleaned in ethanol and IPA, then dried under nitrogen. This preparation stage could be completed in around 2 hours including curing of the epoxy for one hour.

\section{Instrumentation}

The instrument used for sample preparation was an FEI Dual Beam FIB Nova 200, consisting of a $30 \mathrm{kV}$ electron column for scanning electron microscopy (SEM) and a $30 \mathrm{kV}$ sidewinder ion column $\left(\mathrm{Ga}^{+}\right)$, mounted at $52^{\circ}$ to the electron column. The system is equipped with an in-situ micro-manipulator and gas injectors for the in-situ deposition of $\mathrm{Pt}$ and $\mathrm{W}$, which were not required here. Structural characterisation was carried out using a JEOL ARM-200cF scanning transmission electron microscope, using a Gatan Quantum 965 spectrometer for electron energy loss spectroscopy (EELS) analysis. A bespoke detector assembly was used for magnetic characterisation of samples via differential phase contrast imaging, a Lorentz electron microscopy technique [37]. Scanning probe microscopy was performed ex-situ, using a Veeco Dimension 3100 instrument in tapping mode.

\section{FIB Back-milling}

Unlike typical cross-section preparation, a protective Pt layer was not deposited on the sample prior to ion milling because it would be difficult to remove and can damage slightly the uppermost material. Although a protective cap is useful, we tried a variety of materials, including organic coatings that can be subsequently removed by solvents; in each case, the cap obscured visualisation of the thin film in SEM, making it difficult to determine the point at which milling should be stopped. As we show below, if care is taken during the final milling stages, then a protective cap layer is unnecessary. As a consequence, it was essential to avoid imaging or exposing the upper surface of the LSMO to the ion beam at any stage, in order to minimise damage.

The FIB process developed here is outlined in Fig. 1. The first stage, which can be run under the automated control of a script, is to ion-mill a trench of material using a 'polygon' pattern (using the FEI nomenclature [38]). Substrate material was sputtered away by rastering the beam within a user-defined volume, illustrated by the red shaded region in Fig. 1a. Note that in these images, the $\mathrm{Ga}^{+}$beam is oriented normal to the 'top view' and SEM images are collected simultaneously, viewing the sample as sketched in the 'back view'. Thus, the window of material of interest is aligned parallel to the ion beam. This first step was performed using a $30 \mathrm{kV}$ beam and a high beam current, $\mathrm{I}_{\mathrm{ion}}$, of $21 \mathrm{nA}$ in order to remove rapidly a large volume of material. It resulted in a $10 \mu \mathrm{m} \times 20 \mu \mathrm{m}$ thinned region as illustrated. We find that regions wider than $10 \mu \mathrm{m}$ have proved challenging to thin homogeneously and without buckling, although it may be possible to further deepen the regions beyond $20 \mu \mathrm{m}$ or to incorporate additional Pt bars for structural support, as demonstrated elsewhere [39]. An SEM image acquired at the end of the first stage is given in Fig. $1 \mathrm{~b}$ and shows a 
distinctive rectilinear step/terrace morphology within the milled region, which arises from the crystallography of the substrate [40]. The second stage, which can also be automated, continues to thin the region of interest to around $300 \mathrm{~nm}$ (illustrated schematically in Fig. 1c). During the script, the ion beam current was progressively reduced from $6.5 \mathrm{nA}$ for a rough mill, to $2.8 \mathrm{nA}$ and finally to $300 \mathrm{pA}$ for a fine milling stage. Lower beam currents are particularly important for the final stages of milling as they provide sharper probes with smaller tails, thus offering more precise milling and minimising damage. The specimen was tilted to $+1.5^{\circ}$ (i.e. the angle between the $\mathrm{Ga}^{+}$beam and the normal to top view) during the second mill and to $+1.2^{\circ}$ during the fine mill. This increase in incidence angle with respect to the plane of the thinned region increases slightly the sputter yield [41] and acts to smooth or polish the sample face. It is similar to the polishing stage of standard cross-section preparation [10]. An SEM image following this stage is given in Fig. 2d, which shows the back face of the window to be significantly smoother.

The third stage (Fig. 1e,f) thins the region of interest to electron transparency and is best done with live monitoring of the SEM image. A $93 \mathrm{pA}$ beam was used and the specimen was tilted to $+0.2^{\circ}$. Milling was constrained within a rectangular pattern [38] of approximate dimensions $15 \mu \mathrm{m} \times 160 \mathrm{~nm}$. The pixel dwell time was set to ensure that the total patterning time was less than 1 minute, thereby allowing a number of incremental steps with frequent inspection. SEM was used during this stage to observe the changes to the specimen in real time. For example, bending or buckling of the thinned region under the influence of the ion beam may result from accidental irradiation of the front side of the specimen whilst over-polishing would puncture the film itself. Similarly, SEM contrast changes during milling are a good indication of the thickness uniformity and can be assessed in real time, to quickly adapt the milling if inhomogeneities arise.

A final low energy polish was carried out at $5 \mathrm{keV}$ and at an incidence angle of $+7^{\circ}$ to minimise the ion beam damage and $\mathrm{Ga}^{+}$implantation, similar to the final stages of cross-section fabrication. The reduced beam energy is known to reduce damage and implantation, as discussed elsewhere [10]; our own SRIM calculations [42], for example, indicate that the penetration depth at normal incidence of $\mathrm{Ga}^{+}$ions in $\mathrm{SrTiO}_{3}$ reduces from $36 \mathrm{~nm}$ at $30 \mathrm{keV}$ to just $11 \mathrm{~nm}$ at $5 \mathrm{keV}$. An SEM image of the completed window is given in Fig. 1f, where the window appears bright, indicating electron transparency. Indeed, variations in contrast during this stage can be used to ensure that the sample is uniformly thick.

The above FIB protocols take approximately 3 hours, a time that could be scaled in accordance with the thickness of the bulk film, the desired window size and the sputter yield of the materials. The material's sensitivity to damage and $\mathrm{Ga}^{+}$ion implantation may also necessitate a reduction in the ion beam energies during the final milling stage [43], and we have some experience of successful sample preparation using a final $0.5 \mathrm{keV}$ milling step. An attractive aspect of this method is that scripting can be implemented to select a number of locations along the specimen edge in order to mill a series of windows automatically, requiring user intervention only for the final polishing.

\section{Results}

Typical results of the above procedure are illustrated in Fig. 2, which shows a planview section of $\mathrm{La}_{0.7} \mathrm{Sr}_{0.3} \mathrm{MnO}_{3} / \mathrm{STO}(111)$. The bright field TEM image of Fig. 2a demonstrates that relatively large, uniform windows can be fabricated with ease; in 
this case the window is approximately $3 \mu \mathrm{m} \times 5 \mu \mathrm{m}$ in area. Contrast in the image is dominated by thickness fringes and bend contours, which are inevitable in plan-view crystalline samples [44]. They occur here at a relatively low density, in agreement with the thickness and bending that we quantify below. Importantly, the fringes do not prohibit subsequent large area magnetic characterisation by Lorentz TEM. Figs. $2 b$ and $2 \mathrm{c}$ demonstrate that the crystal structure of the specimen has been preserved. The selected area electron diffraction image (Fig. 2b) is dominated by a single set of sharp spots that indicate a lattice spacing of $3.9 \AA$. The pattern lacks either spot splitting arising from recrystallisation or diffuse rings arising from amorphisation: the epitaxial structure has been preserved. Fig. 2c presents direct visualisation of the atomic arrangement, and is a typical high-angle annular dark field (HAADF) image, where the bright spots are individual atom columns, arranged with hexagonal symmetry consistent with viewing the crystal along the $<111>$ axis.

Electron energy loss spectroscopy (EELS) was used to quantify the variations in lamella thickness that were seen as contrast changes in the SEM image of Fig. 1f. Thickness variations arise because the window has a tendency to deflect during milling. Fig. 3a shows a map of sample thickness that has been determined by the spectrum imaging technique [45] whereby low-loss EELS spectra were acquired in each pixel whilst operating the microscope in scanning TEM mode. In this case, a number of spectrum images were stitched together to span the complete field of view. The log-ratio method [46] was then used to determine relative thickness at each pixel, using the mean atomic number of LSMO to derive an electron mean free path [47] and thereby an absolute thickness. These calculations were performed using the Digital Micrograph $^{\mathrm{TM}}$ software package. The results indicate a sample thickness of 69 +/- $25 \mathrm{~nm}$ (mean +/- standard deviation). The standard deviation is dominated by the combination of a thicker region at the top of left of the window and thinner regions running down the right hand side but there are several square microns of material with much better homogeneity. For example, there is a $(4 \mu \mathrm{m})^{2}$ region lying towards the middle of the region where the thickness is $76+/-5 \mathrm{~nm}$ (mean $+/-$ standard deviation). For this particular lamella, we note that the final thickness is less than the nominal $150 \mathrm{~nm}$ of LSMO originally deposited, which is of use for our purposes because we are interested in the properties of LSMO independent of substrate-induced strain. Of course, the technique is equally applicable to thinner epitaxial films with some substrate crystal retained within the window. If very thin specimens are required for nanoanalysis $(<10 \mathrm{~nm})$, the structural stability of large windows may become problematic, and fabricating smaller windows (or smaller regions within windows) may be preferable to maintain mechanical stability. A certain amount of $\mathrm{Ga}^{+}$ion implantation is expected within the underside surface region of the window. For the window described here, a weak Ga L edge at $1115 \mathrm{eV}$ was visible in EELS spectra. Summing the integrated signal across the entire window, we estimate the concentration to be < 1 at.\%: as described below, this level was not found to have any adverse effects on the film properties.

It is common for some buckling and surface roughening of the specimen to occur during FIB milling [48], irrespective of the clamping on three sides of the window. Whilst thickness variations are easy to assess, quantification of surface roughness and buckling is difficult to determine using TEM alone, as images present only a projection of the specimen. Atomic force microscopy (AFM) was therefore used in combination with the above EELS data to assess independently the window topography from thickness variations and results are presented in Fig. 3b. The image 
has been flattened by subtraction of a low-order polynomial to ensure that the unmilled substrate beyond the lamella is flat. The approximate location of the electron-transparent region is indicated by the dashed green line. Notably, the window region was found to be $\sim 120 \mathrm{~nm}$ higher than the surrounding film, with the maximum height found close to the centre of the window. This bulging may be surprising given the initial tensile epitaxial strain caused by a $\sim 1 \%$ lattice mismatch between the STO substrate (lattice constant, $a=0.391 \mathrm{~nm})$ and LSMO film $(a=0.387 \mathrm{~nm})$ but it should be noted that at a film thickness of $120 \mathrm{~nm}$, the lattice mismatch will have been accommodated by misfit dislocations and that the film will be largely strain-free. Indeed, although the buckling looks substantial by AFM, it represents an extension of less than $0.03 \%$ across the $\sim 11.7 \mu \mathrm{m}$ lamella width. We are currently exploring results in systems with a greater amount of residual strain as the preparation technique outlined here provides a useful means of manipulating the in-plane strain applied to films, which could be useful in studies of functionality including magnetism. We find it common for lamellae to buckle slightly during milling, resulting in a thicker central region that can be compensated for by changing the milling pattern. It is also interesting to note that the buckling in this case extends beyond the electron transparent region because the final milling stage created a 'pocket' behind the lamella that is also evident in the SEM image of Fig. 1f and which could be removed by reducing the duration of the final milling stage. Ideally, pocket formation should be minimised.

AFM also allows the surface roughness, and hence damage, to be assessed directly. Representative line-scans taken from the middle of the upperside of the lamella (light green trace) and from a region that has not been exposed to the $\mathrm{Ga}^{+}$beam (dark blue trace) are presented in figure Fig. $3 \mathrm{c}$. Both line traces were collected from a $(5 \mu \mathrm{m})^{2}$ scan and have had a low-order polynomial subtracted to isolate high spatial frequencies from scan distortions and from the buckling effect. We find that the peakto-peak roughness is increased slightly on the window surface, which we attribute to slight modification and sputtering caused by imaging with the ion beam at low incidence angles for registration during the script. It is also likely that the line trace collected away from the window was compromised by surface contamination and residue from the epoxy and wax used during the sample preparation process, since atomic-scale steps were not observed but have been measured on similar surfaces after appropriate cleaning. Nevertheless the modest alteration to film roughness indicated by Fig. $3 \mathrm{c}$ did not cause any perceptible changes to the film properties.

With the thickness map presented above, it is possible to derive an absolute measure of the LSMO film's (in-plane) magnetic induction using Lorentz microscopy techniques [37], and thereby measure the Curie temperature of the LSMO within the window region. A detailed magnetic characterisation of the film is beyond the scope of this publication and will be reported separately. However, Fresnel images are given in Fig. 4 to illustrate that the film is ferromagnetic at room temperature. Fresnel imaging is performed by conventional TEM, and reveals magnetic contrast when the imaging lens is defocused. This has the effect of raising or lowering the object plane relative to the specimen, depending on whether the lens is under- or over-focused; no magnetic contrast is observed when the image is in focus. Fig. 4a shows an underfocused Fresnel image, which highlights a magnetic domain wall in the form of a bright band of electron intensity, indicated by the red arrows. Confirmation that this is magnetic contrast may be obtained by observing a focused image of the same region, as in Fig. 4b, where the magnetic domain wall disappears. By defocusing the 
imaging lens in the opposite direction, Fig. 4c, the domain wall appears with the reverse contrast, i.e. a dark band of intensity. The main result of the Lorentz characterisation is that the local Curie temperature is hardly affected by the process of making a plan-view specimen. For example, the Curie temperature of the film within a similar window to that illustrated here was found to be $349 \pm 3 \mathrm{~K}$, which is in excellent agreement with the as-grown value of $353 \mathrm{~K}$. This result is important because both damage and Ga implantation [34] impair $\mathrm{T}_{\mathrm{c}}$ in LSMO: the $\mathrm{T}_{\mathrm{c}}$ recorded here therefore indicates very little damage.

\section{Summary and conclusions}

In summary, we have detailed a simple, practical method for fabricating large area plan-view TEM specimens, with minimal damage. Specific milling parameters have been provided for each stage, aiding application of the technique to other material systems. The technique's main novelty is its simplicity as it is fast, and does not involve complex material pre-treatment or micromanipulation. The technique is sitespecific and ideally suited to the production of large plan-view areas of epitaxial thin films with a reasonable yield. It can be readily applied to other material systems by appropriate consideration of ion beam energies and milling parameters. Furthermore, it is feasible to create a large number of plan-view samples from one substrate, or to mill a series of windows along one edge of a specimen, separated by un-milled bars for structural support. This technique therefore offers the ability to view large regions of a specimen in plan-view that would otherwise be challenging with conventional methods.

\section{Acknowledgements}

This work was funded by EU's $7^{\text {th }}$ framework Program IFOX (NMP3-LA-2010 246102) and the Engineering and Physical Sciences Research Council of the UK (EP/I00419X/1).

\section{References}

[1] Giannuzzi, L. A., Stevie, F. A., 1999. A review of focused ion beam milling techniques for TEM specimen preparation, Micron 30, 197.

[2] Mayer, J., Giannuzzi, L. A., Kamino, T., Michael, J., 2007. TEM sample preparation and FIBinduced damage, MRS Bulletin 35, 400.

[3] Li, J., Malis, T., Dionne, S., 2006. Recent advances in FIB-TEM specimen preparation techniques, Materials Characterization 57, 64.

[4] Chappert, C., Bernas, H., Ferré, J., Kottler, V., Jamet, J.-P., Chen, Y., Cambril, E., Devolder, T., Rousseaux, F., Mathet, V., Launois, H., 1998. Planar patterned magnetic media obtained by ion irradiation, Science 280, 1919.

[5] McGrouther, D., McVitie, S., Chapman, J. N., Gentils, A., 2007. Controlled domain wall injection into ferromagnetic nanowires from an optimized pad geometry, Appl. Phys. Lett. 91, 022506.

[6] Vick, D., Sauer, V., Fraser, A. E., Freeman, M. R., Hiebert, W. K., 2010. Bulk focused ion beam fabrication with three-dimensional shape control of nanoelectromechanical systems, J. Micromech. Microeng. 20, 105005. 
[7] Basith, M. A., McVitie, S., McGrouther, D., Chapman, J. N., 2012. Reproducible domain wall pinning by linear non-topographic features in a ferromagnetic nanowire, Appl. Phys. Lett. 100, 232402.

[8] Kaminsky, W. M., Jones, G. A. C., Patel, N. K., Booij, W. E., Blamire, M., Gardiner, S. M., Xu Y. B., Bland, J. A. C., 2001. Patterning ferromagnetism in Ni80Fe20 films via $\mathrm{Ga}^{+}$ion irradiation, Appl. Phys. Lett. 78, 1589.

[9] Woods, S. I., Ingvarsson, S., Kirtley, J. R., Hamann, H. F., Koch, R. H., 2002. Local magnetic anisotropy control in NiFe thin films via ion irradiation, Appl. Phys. Lett. 81, 1267.

[10] Schaffer, M., Schaffer, B., Ramasse, Q., 2012. Sample preparation for atomic-resolution STEM at low voltages by FIB, Ultramicroscopy 114, 62 .

[11] Rivera, F., Davis, R., Vanfleet, R., 2013. Alternative FIB TEM sample preparation method for cross-sections of thin metal films deposited on polymer substrates, Microsc. Microanal. 19, 1080.

[12] Grandfield, K., Engqvist, H., 2012. Focused Ion Beam in the Study of Biomaterials and Biological Matter, Advances in Materials Science and Engineering 2012, 841961.

[13] Zhang, W., Theil Kuhn, L., Jørgensen, P. S., Thydén, K., Bentzen, J. J., Abdellahi, E., Sudireddy, B. R., Chen, M., Bowen, J. R., 2013. Transmission electron microscopy specimen preparation method for multiphase porous functional ceramics, Microsc. Microanal. 19, 501.

[14] Langford, R. M., Petford-Long, A. K., 2001. Preparation of transmission electron microscopy cross-section specimens using focused ion beam milling, J. Vac. Sci. Technol. A 19, 2186.

[15] Yi, G., Nicholson, W. A. P., Lim, C. K., Chapman, J. N., McVitie, S., Wilkinson, C. D. W., 2004. A new design of specimen stage for in situ magnetising experiments in the transmission electron microscope, Ultramicroscopy 99, 65.

[16] Yao, B., Petrova, R. V., Vanfleet, R. R., Coffey, K. R., 2006. A modified back-etch method for preparation of plan-view high-resolution transmission electron microscopy samples, J. Elec. Microsc. 55, 209.

[17] Yao, B., Coffey, K. R., 2008. Back-etch method for plan view transmission electron microscopy sample preparation of optically opaque films, J. Elec. Microsc. 57, 47.

[18] Eberg, E., Monsen, A. F., Tybell, T., van Helvoort, A. T. J., Holmestad, R., 2008. Comparison of TEM specimen preparation of perovskite thin films by tripod polishing and conventional ion milling, J. Elec. Microsc. 57, 175.

[19] McCaffrey, J. P., Phaneuf, M. W., Madsen, L. D., 2001. Surface damage formation during ionbeam thinning of samples for transmission electron microscopy, Ultramicroscopy 87, 97.

[20] Voyles, P., Muller, D., Grazul, J., Citrin, P., Gossmann, H.-J., 2002. Atomic-scale imaging of individual dopant atoms and clusters in highly n-type bulk Si, Nature 416, 826.

[21] Kirk, K. J., Chapman, J. N., Wilkinson, C. D. W., 1999. Lorentz microscopy of small magnetic structures, J. Appl. Phys. 85, 5237 .

[22] Gu, E., Bland, J. A. C., Daboo, C., Gester, M., Brown, L. M., Ploessl, R., Chapman, J. N., 1995. Microscopic magnetization reversal processes and magnetic domain structure in epitaxial Fe/GaAs(001) films, Phys. Rev. B 51, 3596.

[23] Floresca, H. C., Jeon, J., Wang, J. G., Kim, M. J., 2009. The focused ion beam fold-out: sample preparation method for transmission electron microscopy, Microsc. Microanal. 15, 558.

[24] Mayer, J., Giannuzzi, L. A., Kamino, T., Michael, J., 2007. TEM sample preparation and FIB induced damage, MRS Bulletin 32, 400.

[25] Patterson, R. J., Mayer, D., Weaver, L., Phaneuf, M. W., 2002. "H-Bar Lift-Out" and "PlanView Lift-Out”: Robust, Re-thinnable FIB-TEM Preparation for Ex-Situ Cross-Sectional and Plan-View FIB Specimen Preparation, Microsc. Microanal. 8, 566.

[26] Stevie, F. A., Irwin, R. B., Schofner, T. L., Brown, S. R., Drown, J. L., Giannuzzi, L. A., 1998. Plan view TEM sample preparation using the focused ion beam lift-out technique, AIP Conf. Proc. 449, 868 . 
[27] Schreiber, D. K., Adusumilli, P., Hemesath, E. R., Seidman, D., Petford-Long, A. K., Lauhon, L. J., 2012. A method for directly correlating site-specific cross-sectional and plan-view transmission electron microscopy of individual nanostructures, Microsc. Microanal. 18, 1410.

[28] Tsutsumi, T., Suzuki, E., Ishii, K., Kanemaru, S., Maeda, T., Tomizawa, K., 1999. Plane-view observation technique of silicon nanowires by transmission electron microscopy, J. Vac. Sci. Technol. B 171897.

[29] Langford, R. M., Huang, Y. Z., Lozano-Perez, S., Titchmarsh, J. M., Petford-Long, A. K., 2001. Preparation of site specific transmission electron microscopy plan-view specimens using a focused ion beam system, J. Vac. Sci. Technol. A 19, 2186.

[30] Langford, R. M., 2006. Focused ion beams techniques for nanomaterials characterization, Microscopy Research and Technique 69, 538.

[31] Fox, D., Verre, R., O’Dowd, B. J., Arora, S. K., Faulkner, C. C., Shvets, I. V., Zhang, H., 2013. Investigation of coupled cobalt-silver nanoparticle system by plan view TEM, Progress in Natural Science: Materials International, 23, 588.

[32] Vrejoiu, I., Alexe, M., Hesse, D., Gosele, U., 2008. Functional Perovskites - From Epitaxial Films to Nanostructured Arrays, Adv. Funct. Mater. 183892.

[33] Ramesh R., Spaldin, N., 2007. Multiferroics: progress and prospects in thin films, Nat. Mater., 6, 21.

[34] Hao, C., Zhao, B., Kuang, G., Sun, Y., 2011. Comparing study of magnetic-ion and nonmagnetic-ion doping effect at $\mathrm{Mn}$ site in $\mathrm{La} 0.825 \mathrm{Sr} 0.175 \mathrm{MnO} 3$ compound, Phys. Status Solidi B 248, 2921.

[35] Moshnyaga, V., Khoroshun, I., Sidorenko, A., Petrenko, P., Weidinger, A., Zeitler, M., Rauschenbach, B., Tidecks R., Samwer, K., 1999. Preparation of rare-earth manganite-oxide thin films by metalorganic aerosol deposition technique, Appl. Phys. Lett. 74, 2842.

[36] Chakhalian, J., Millis, A. J., Rondinelli, J., 2012. Whither the oxide interface, Nature Materials 11, 92.

[37] Chapman, J. N., 1984. The investigation of magnetic domain structures in thin foils by electron microscopy, J. Phys. D: Appl. Phys. 17, 623.

[38] FEI Company, xT Nova NanoLab User’s Manual, 2006, fifth ed., p55.

[39] Jublot, M., Texier, M., 2014. Sample preparation by focused ion beam micromachining for transmission electron microscopy imaging in front-view, Micron 56, 63.

[40] Ishitani, T., Yaguchi, T., 1996. Cross-sectional sample preparation by focused ion beam: A review of ion-sample interaction, Microsc. Res. Tech. 35, 320.

[41] Adams, D. P., Vasile, M. J., Mayer, T. M., 2006. Focused ion beam sculpting curved shape cavities in crystalline and amorphous targets, J. Vac. Sci. Technol. B 24, 1766.

[42] SRIM software by J. F. Ziegler freely available from www.srim.org.

[43] Kato, N. I., 2004. Reducing focused ion beam damage to transmission electron microscopy samples, J. Electron Microsc. 53, 451.

[44] Williams, D. B., Carter, C. B., 1996. Transmission Electron Microscopy, pp. 369-378, Springer.

[45] Jeanguillaume, C., Colliex, C., 1989. Spectrum-image: The next step in EELS digital acquisition and processing, Ultramicroscopy $28,252$.

[46] Egerton, R. F., 2011. Electron Energy-Loss Spectroscopy in the Electron Microscope, 3rd ed., Springer.

[47] Malis, T., Cheng, S. C., Egerton, R. F., 1988. EELS log-ratio technique for specimen-thickness measurement in the TEM, J. Electr. Microsc. Technol., 8, 193.

[48] Jin, H.-H., Shin, C., Kwon, J., 2010. Fabrication of a TEM sample of ion-irradiated material using focused ion beam microprocessing and low-energy Ar ion milling, J. Electron Microsc. 59, 463. 


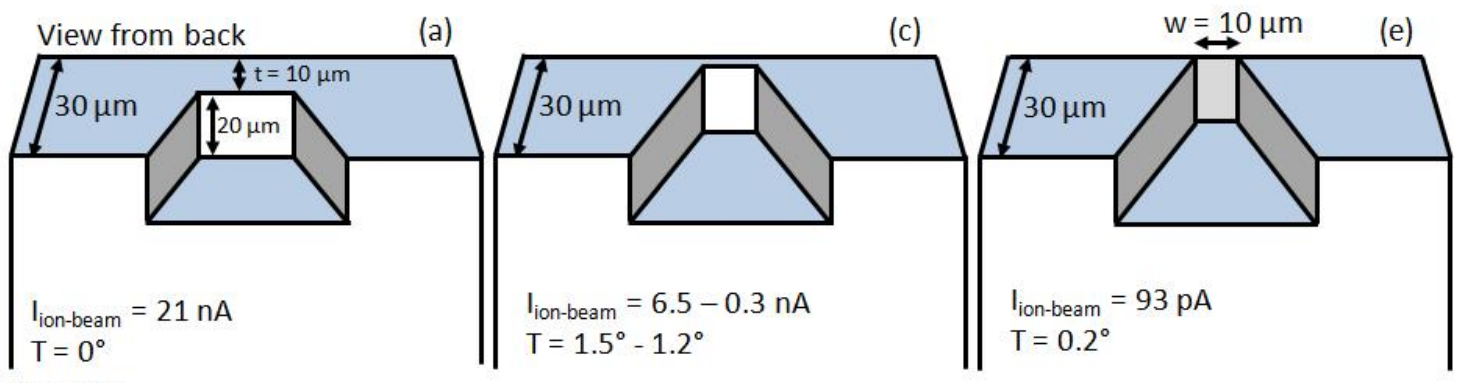

Top view
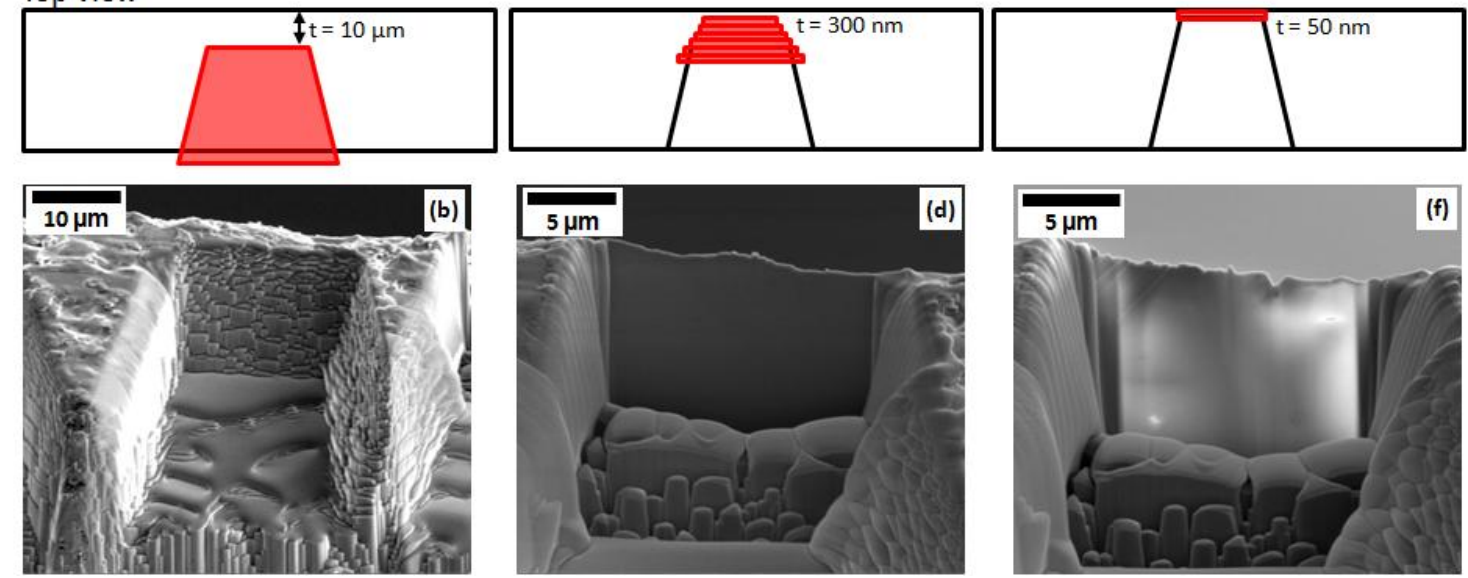

FIG. 1: (a,c,e) Schematic illustration of successive stages of window fabrication with (b,d,f) corresponding SEM images. Upper panels illustrate the SEM view whilst middle panels ('top view') are as seen from the ion column, with the ion beam rastering within the red sections. The first stage $(a, b)$ uses a beam current of $21 \mathrm{nA}$ to achieve a window thickness of $10 \mu \mathrm{m}$; the second stage (c,d) uses beam currents from $6.5 \mathrm{nA}$ to $0.3 \mathrm{nA}$ to achieve a window thickness of $300 \mathrm{~nm}$; and the third stage uses an ion beam current of $93 \mathrm{pA}$ to achieve electron transparency. 


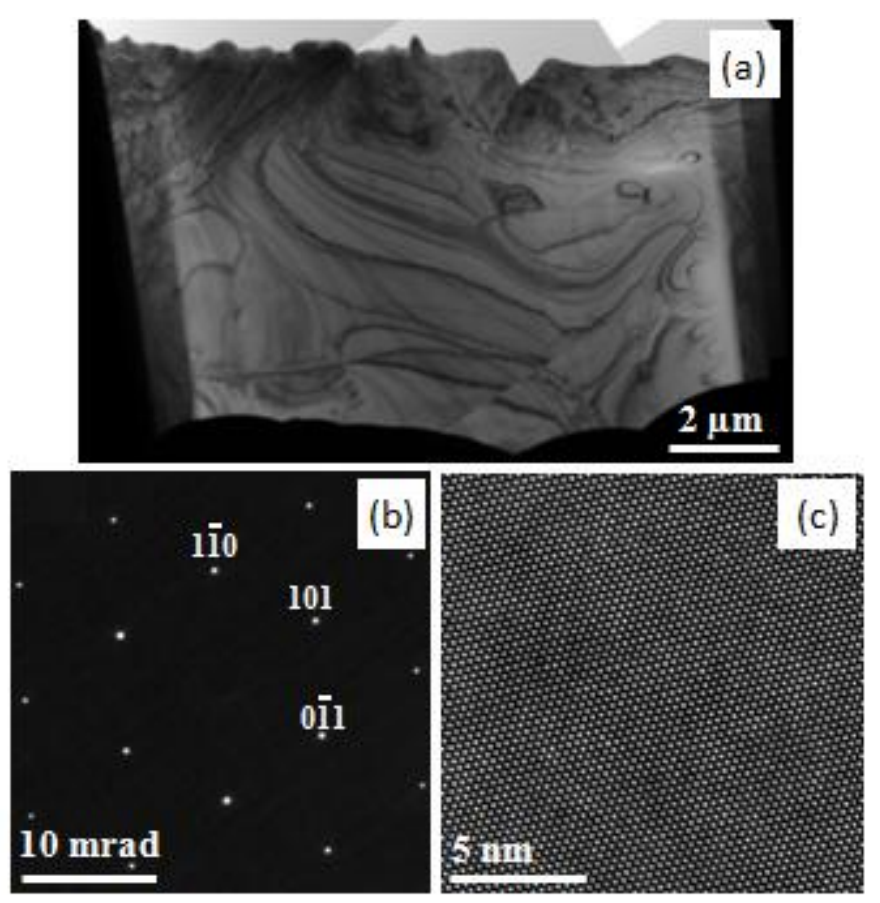

FIG. 2: (a) Bright field image of the electron transparent window region, composed of several bright field images stitched together. (b) A selected area diffraction pattern showing sharp spots with hexagonal symmetry and no apparent structural changes. (c) High-angle annular dark field image of a region close to the centre of the film viewed along the <111> axis, confirming preservation of the crystal structure. 

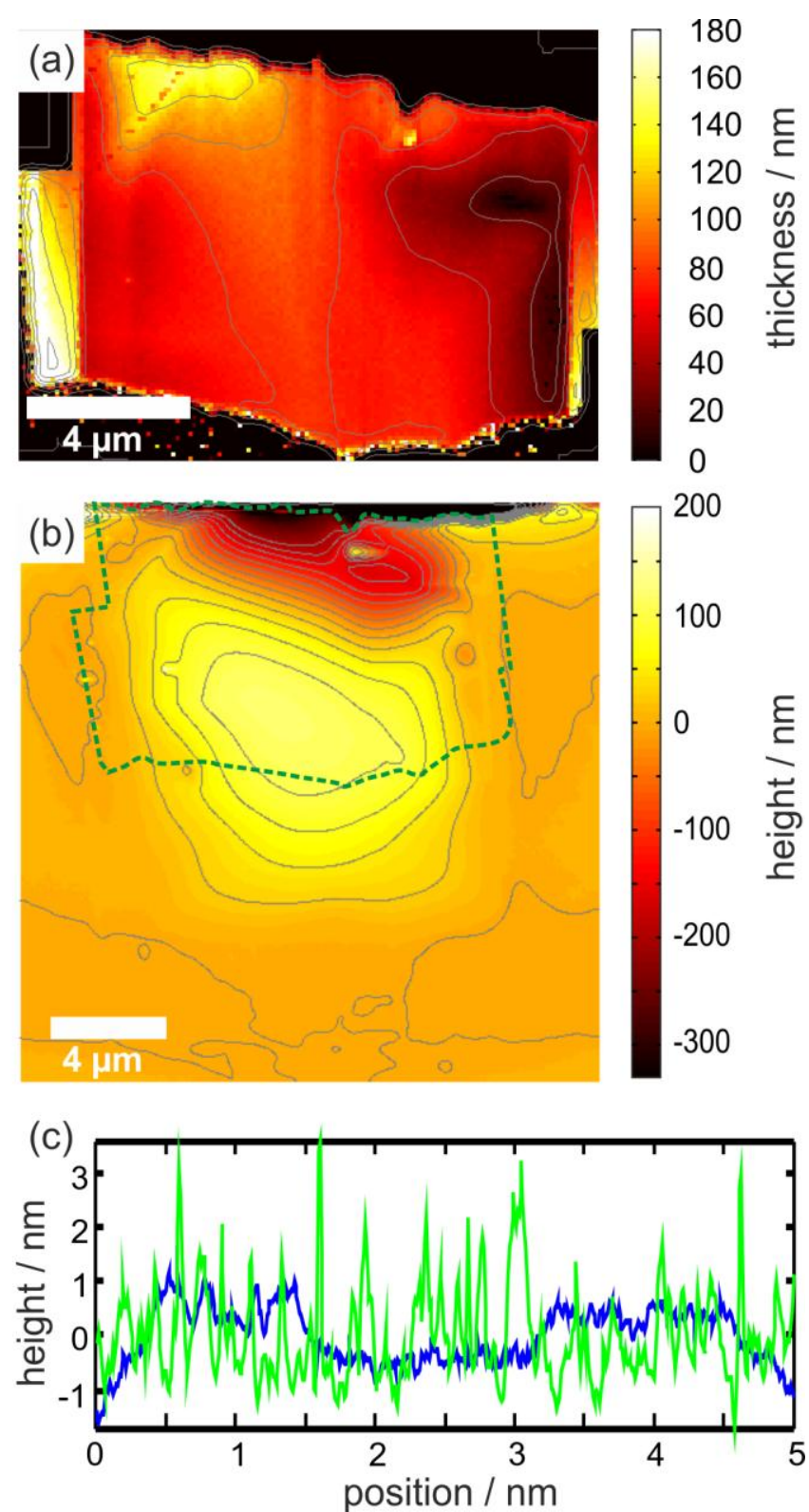

FIG. 3: (a) Thickness map determined by electron energy loss spectroscopy (EELS) with contours to guide the eye. (b) AFM height map of the window region, with the approximate extent of the electron-transparent region indicated by the green dotted line. The image has had a low-order polynomial subtracted to remove scan distortions and thereby flatten the surface beyond the window. The window is buckled by up to $120 \mathrm{~nm}$ with respect to the unmilled substrate. (c) Typical AFM line traces across the surface of the window (light green) away from the window (dark blue), again after removal of a low-order polynomial. 


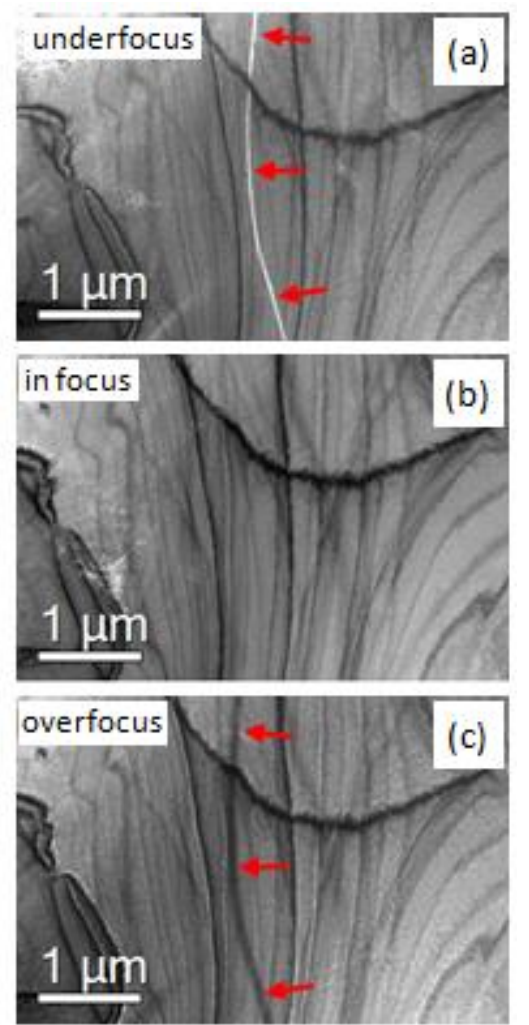

FIG. 4: Fresnel image sequence at (a) underfocus, (b) in focus and (c) overfocus. The domain wall indicated by the red arrows in (a) appears bright; this contrast reverses for an overfocused image, where the domain wall appears dark. 